\title{
Fiber ICAN laser with exawatt-picosecond pulses for fusion without nuclear radiation problems
}

\author{
H. HORA, ${ }^{1}$ P. LALOUSIS, ${ }^{2}$ AND S. MOUSTAIZIS ${ }^{3}$ \\ ${ }^{1}$ Department of Theoretical Physics, University of New South Wales, Sydney, Australia \\ ${ }^{2}$ Institute of Electronic Structure and Laser FORTH, Heraklion, Crete, Greece \\ ${ }^{3}$ Technical University of Crete, Chania, Greece
}

(RECEIVED 19 September 2013; ACCEPTED 20 September 2013)

\begin{abstract}
One of the numerous applications of the ICAN laser using the advantage of fiber optics with chirped pulse amplification (CPA), is the scheme of side-on initiation of a nuclear fusion flame in solid density fuel with laser pulses of shorter than picosecond (ps) duration and power in the petawatt (PW) and higher range. The ICAN Fiber optics has special advantages with the potential that $>900 \mathrm{PW}$ spherical laser pulses may ignite the proton reaction with ${ }^{11} \mathrm{~B}$ (HB11) without the problem of dangerous radioactive radiation. Though secondary reactions can be estimated very roughly, the feasibility of a power station with the necessary energy gains can be concluded.
\end{abstract}

Keywords: Clean nuclear energy; Exawatt pulses; Fiber-laser; Laser driven fusion; Proton-11boron fuel

\section{INTRODUCTION}

Fiber glass lasers for amplification of pulses of lower than picosecond (ps) duration and powers of petawatt (PW) up to exawatt (EW) and higher by the ICAN project using chirped pulse amplification (CPA) (Mourou et al., 2013) are of interest for electron or hadron accelerators with many applications including cancer therapy, as well as for generating PeV particles or high energy physics for exploring extremely high field processes as pair production in vacuum. Another application is for nuclear fusion for generating energy where the problem of the dangerous radioactive radiation of all presently developed nuclear energy schemes can be reduced below the radiation from burning coal, i.e., below any dangerous level. The scheme "has the potential to be the best route to fusion energy" (Haan, 2010) and is one of the applications opened by the CPA discovery (Strickland et al., 1985) which led to the CPA-ICAN lasers (Mourou et al., 2013).

This application for laser driven fusion was not easy to be opened and because this was realized only on a rather narrow line of research though the principals involved with the nonlinearity of laser physics have a long tradition. The ICAN technique provides very favorable conditions for this

Address correspondence and reprint requests to: H. Hora, Department of Theoretical Physics, University of New South Wales, Sydney 2052, Australia.E-mail: h.hora@unsw.edu.au application for fusion as it will be adding to the groundbreaking new aspects (Mouroou et al., 2013). The key measurement is the experimental confirmation of the ultrahigh acceleration using the very short laser pulses with the very high power by CPA, first measured by Sauerbrey (1996) when using extremely high contrast ratios to cut-off laserprepulses by a higher ratio than $10^{7}$ until a time of about 1 ps before the main laser pulse arrived at the target. The repetition of this experiment was possible only by using the extreme quality of laser pulses by Földes et al. (2000), while any slight reduction of the quality prevented the measurement of this effect. This was clarified by the teams of Zhang et al. (1998), Badziak et al. (1999), and Klaus Witte (Kaluza et al., 2004).

The measured ultrahigh acceleration (Sauerbrey, 1996) of $2 \times 10^{20} \mathrm{~cm} / \mathrm{s}^{2}$ was 100,000 times higher than ever measured before in a laboratory. This extremely high acceleration was predicted numerically in 1978 (see Hora, 1981) in details (Hora et al., 2007) as nonlinear-force (ponderomotive) collective acceleration of plasma blocks. On top, the discovery of ultrahigh ion current densities in the space charge neutral accelerated plasma blocks above $10^{13} \mathrm{Amps} / \mathrm{cm}^{2}$ is more than million times higher than can produce accelerators (Hora et al., 2002; Badziak et al., 2004).

After considering the new aspects of the fiber option (Mourou et al., 2013) for PW to EW and more powerful laser pulses, we report on laser fusion of uncompressed 
solid density deuterium-tritium (DT) fuel at spherical irradiation where the properties can realistically followed up on numerous confirming preceding computations. The use of solid density HB11 fuel resulted in similar gains when using the same presumptions as for DT (Hora, 2009; Hora et al., 2009). This was not including the secondary reactions of the generated alpha particles when hitting protons leading to an avalanche of reactions with an essentially much higher total fusion gain, even to higher values than with DT. One may note, that the HB11 reaction is then the first to realize a neutron-free reaction (Tahir et al., 1997; Labaune et al., 2013), which the highly supported DT fusion schemes cannot provide, for which the classical approach has not arrived at the break-even level yet. The generated neutrons for the DT reaction decay with a half-life of 12 min into protons and electrons to produce water but before reaching this, the neutrons can react with stable nuclei to produce large amounts of radioactivity. These problems are eliminated with the neutron-free HB11 reaction.

Without the availability of details of the essential secondary reactions of the alpha particles (Hora et al., 2012), an evaluation of estimations for the fusion gain is possible for the conditions of irradiating spheres of solid density HB11 fuel by converging ICAN laser pulses.

\section{BENEFITS OF THE FIBER ICAN}

The application to nuclear fusion was based on the ChuBobin scheme (Chu, 1972; Bobin, 1974) of side-on ignition of a fusion flame in uncompressed solid density deuteriumtritium where it was shown that the flame can be initiated by a ps energy input into the fuel having an energy flux above $10^{8} \mathrm{~J} / \mathrm{cm}^{2}$. It was completely impossible to be realized with the lasers in 1972 and the thermal compression and ignition of the fusion fuel by nanosecond laser pulses only could be followed up using indirect drive (Lind et al., 2011; Haan et al., 2011), fast ignition (Azechi et al., 2011), or direct drive (Hora et al., 1998; Hora, 2013). However, when using CPA (Sauerbrey, 1996; Hora et al., 2002; Hora, 2004) the unexpected conditions of the Chu-Bobin scheme could be fulfilled for the side-on ignition of uncompressed solid density fuel. This was initially evaluated for DT

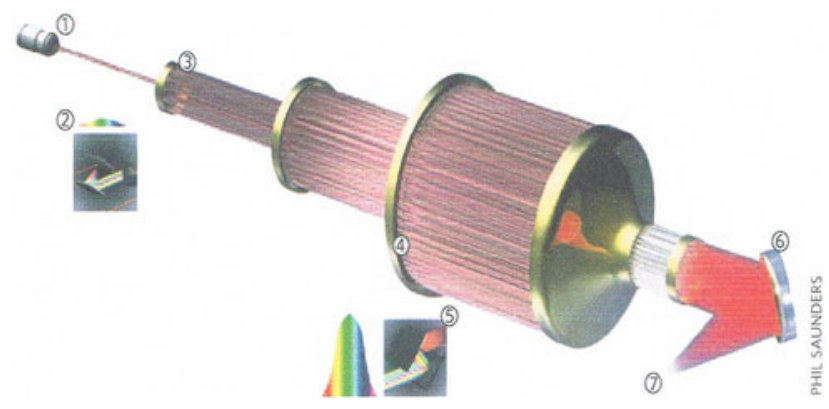

Fig. 1. (Color online) ICAN laser (Mourou et al., 2013).

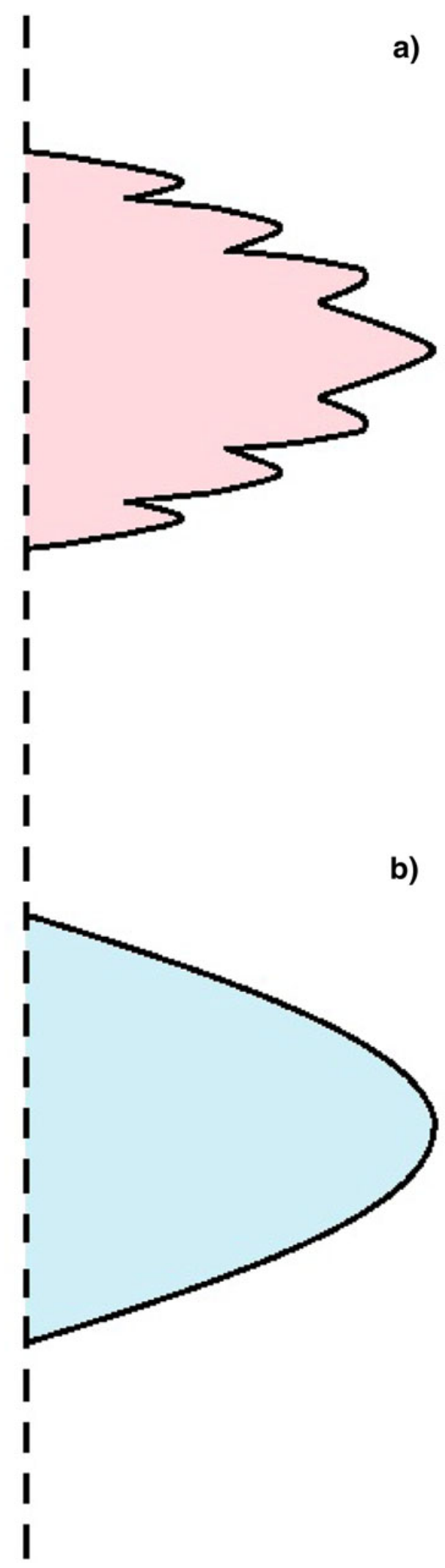

Fig. 2. (Color online) Intensity distribution (schematic) across the laser beam after amplification (a) from a solid state laser (upper part) with irregular hot spots (maxima) in contrast to (b) the ideal output from an ICAN fiber laser (Mourou, 2013; Mourou et al., 2013). 
fusion fuel for irradiating laser pulses in plane geometry (Hora, 2009).

For a fusion power station, problems of lateral energy losses of the plane irradiation can be overcome by using spherical irradiation. For this case, the ICAN fiber laser offers a solution as can be seen from Figure 1 (Mourou et al., 2013). The output of the amplified beam before the focusing of mirror 6 is from parallel fibers and has a plane wave front. In practical cases, the beam of $100 \mathrm{~cm}^{2}$ cross-section may be a pulse with $1 \mathrm{~kJ}$ energy and of ps duration representing $1 \mathrm{PW}$. The wave front is of such a quality that focusing in 7 has been shown (Mourou et al., 2013) to go to a diameter of $10 \mu \mathrm{m}$ such that intensities at about $10^{21} \mathrm{~W} / \mathrm{cm}^{2}$ are reached. The fibers permit an exceptionally high quality of the beam uniformity as it was shown in Figure 2 (Mourou, 2013) where the usual undesired maxima in the beam profile with glass lasers are automatic eliminated due to the fiber optics quality.

A further advantage of the fiber optics is by avoiding the focusing mirror 6 in Figure 1, when taking the axes at the ends of the fibers not parallel at the end. Instead of the plane wave fronts from parallel axes of the fibers in Figure 1, the axes should be directed radial to a spherical center 2 as shown in Figure 3 such that a whole sphere 1 produces a spherical converging laser pulse front towards the center 3 where a fusion fuel pellet 3 is located. The fiber ends should fill nearly completely the whole sphere 1 . If the radius of 1 is $1 \mathrm{~m}$ or more, a converging laser pulse with 1 ps duration and a power of more than EW will be generated for hitting the fuel pellet 2 . Based on kJ energy per $100 \mathrm{~cm}^{2}$ output from a sphere of about $2 \mathrm{~m}$ diameter, a spherical laser pulse of EW and 1 ps duration would be generated.

\section{RESULTS WITH DEUTERIUM-TRITIUM FUEL}

In order to understand the mechanisms in the spherically irradiated solid density fuel, results from computations using the genuine two-fluid hydrodynamics are being shown. In

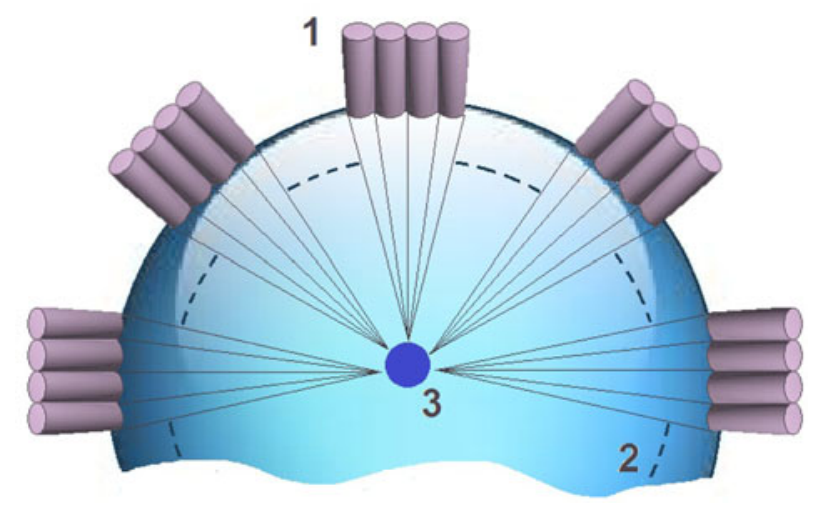

Fig. 3. (Color online) Generation of a spherical shrinking laser pulse from radially directed fiber ends at a radius 1 to irradiate a spherical solid state fusion fuel 3 with ta concentric grid 2 of positive electric charge for slowing down the generated alpha particles from the HB11 reactions. this model case, the radius of the pellet is taken $0.5 \mathrm{~mm}$ similar to the cases evaluated before (Moustaizis et al., 2013) with a laser intensity of $10^{20} \mathrm{~W} / \mathrm{cm}^{2}$ for the initiating ps laser pulse. Figure 4 shows the ion density $n_{\mathrm{i}}$ within the sphere at a sequence of times, Figure 5 the fusion reaction rate and Figure 6 the electron temperature. The main push is in the radial directed ions while the complete hydrodynamic code shows the highly delayed heating of the electrons in the collectively accelerated plasma blocks in Figure 6.

The conditions for the numerical evaluation of the initiation of the fusion flame by ultrahigh acceleration of nonlinear force driven plasma blocks by non-thermal direct conversion of laser energy into the collective driving process involves the result of Chu (1972) that the energy flux density $E^{*}$ threshold for the picosecond laser pulses of $4 \times 10^{8} \mathrm{~J} / \mathrm{cm}^{2}$ has to be reduced by a factor 20 (Hora et al., 2008) for DT to

$$
E^{*}=2 \times 10^{7} \mathrm{~J} / \mathrm{cm}^{2} .
$$

The reason for this reduction is the fact that at the time of the work of Chu, the inhibition factor for the reduction of thermal conduction in inhomogeneous plasmas was not known and the collective stopping power of Denis Gabor was not used as needed for high density plasmas. The experimental confirmation of these processes was shown while the quantum correction of the collision frequency is important only for longer times than the ps interactions (Hora et al., 2012).

The collective block acceleration could best be seen, e.g., from the very low energy of the space charge neutralizing electrons between the directed motion of the blocks where the main energy is in the ions. The computations with the general hydrodynamic code (Lalousis, 1983; Hora et al., 1984; 2013; Hora, 2011; Lalousis et al., 2012; 2013) include the separate temperatures of the electrons and of the ions

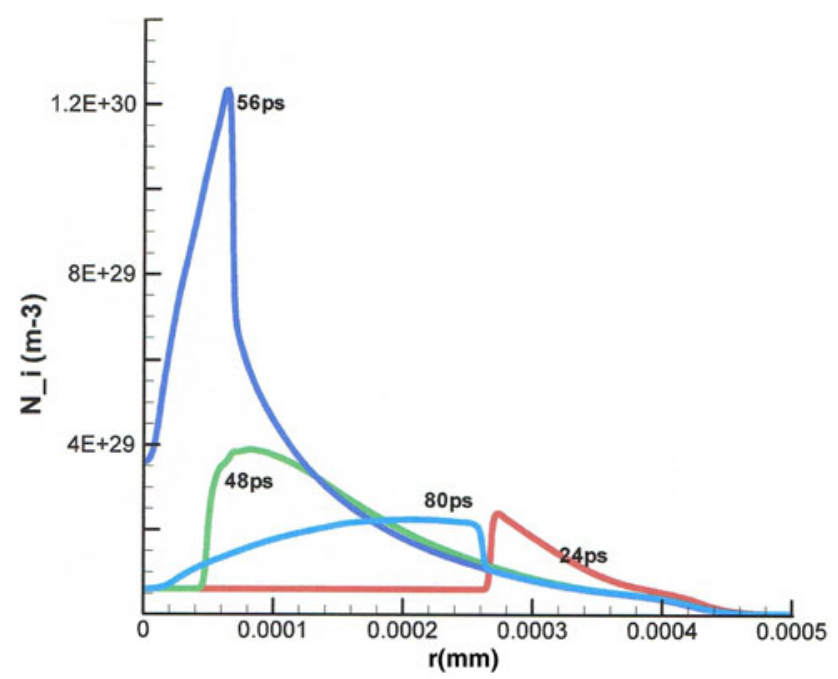

Fig. 4. (Color online) Initiation of a fusion flame in a solid DT sphere of $0.5 \mathrm{~mm}$ radius in the center of Figure 3 irradiated by a 1 ps laser pulse of $10^{20} \mathrm{~W} / \mathrm{cm}^{2}$. The profile of the ion density is shown at different time after the initiating ps laser pulses deposited the energy to the driving plasma block. 


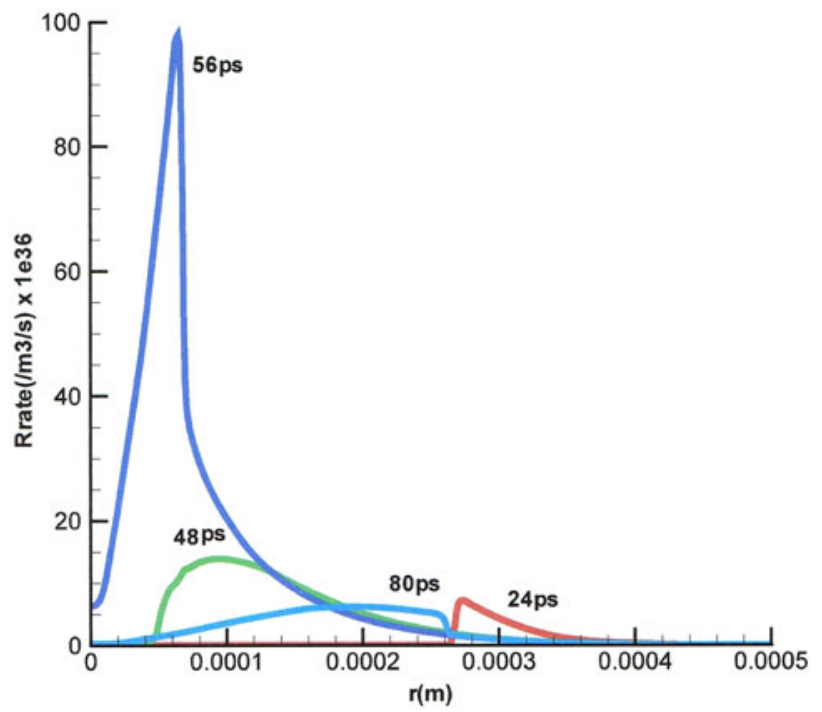

Fig. 5. (Color online) The reaction rate for the case of Figure 4 .

based on the genuine two-fluid model with complete inclusion of collisions in contrast to the initial computations (Chu, 1972) with modifications (Malekynia et al., 2013). The stopping power for the generated charged nuclear products was a first option based on the same collective model as for DT (Hora, 2009; Hora et al., 2009).

\section{GENERATION OF A SPHERICAL FUSION FLAME FOR IGNITION OF HYDROGEN BORON}

The stopping power for the generated charged nuclear products was as first option based on the same collective model as for DT (Hora, 2009; Hora et al., 2009). This is in strong contrast to the fusion of HB11 though it was very helpful that the nonlinear force driven block ignition of the

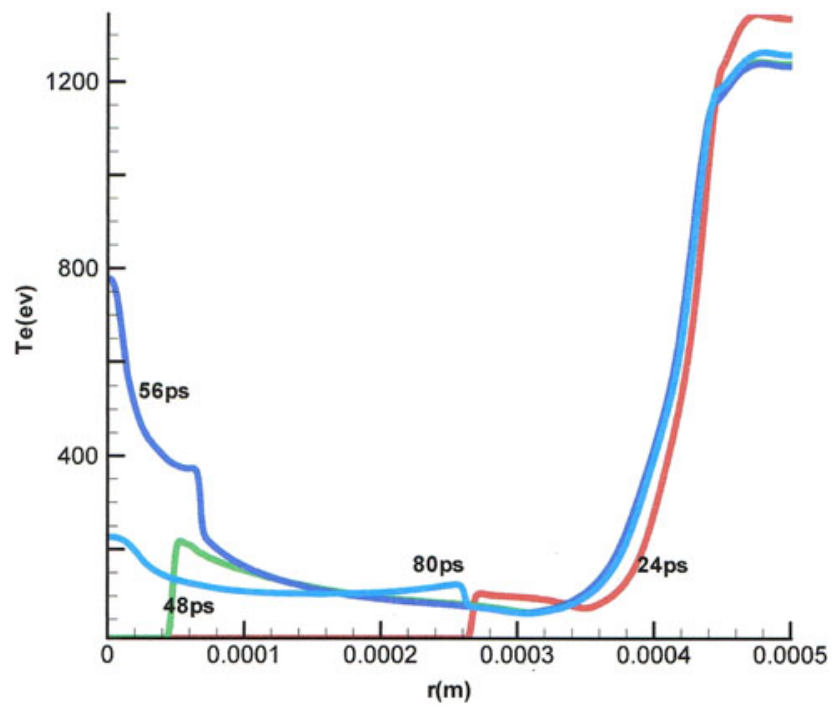

Fig. 6. (Color online) The electron temperature from the slowly thermalizing of the directed ion energy of the nonlinear force accelerated plasma blocks. fusion flame by picoseconds laser pulses led to fusion gains about 5 times more complicate only than for DT. With thermal nanosecond laser pulse driving to the fusion, HB11 was about 100,000 times more difficult (Stening et al., 1992) and relaxed to a factor 1,000 due to a resonance process (Kouhi et al., 2011).

It was then rather a surprise that computations of the fusion flame ignition of HB11 was only less than about 5 times more difficult than with DT fuel even with the pessimistic assumption that the stopping of the generated $2.89 \mathrm{MeV}$ alpha particles was described by the collective electron interaction only, neglecting the enormous multiplication process of secondary alpha generation (Hora et al., 2012a). This is important when considering the nuclear fusion of a sphere 3 in the center of Figure 3 of solid density HB11.

Based on the reaction

$$
p+{ }^{11} B=3{ }^{4} \mathrm{He}+8.9 \mathrm{MeV},
$$

where the resulting helium nuclei (alpha particles) have each the same energy of $2.89 \mathrm{MeV}$, the simplified assumption for the stopping power (Hora et al., 2009) neglected the reaction by the pre-cursing alpha particles beyond the shock (Hora et al., 2011a) of the fusion flame and used only stopping by electrons resulting in higher gains. As has been shown (Hora et al., 2012a; Moustaizis et al., 2013) when discussing spherical irradiation geometry, an additional stopping is due to alpha-proton and alpha-boron collisions. This is not only important for shorter stopping lengths but the energy transferred from the alpha particles to protons is in the range of $600 \mathrm{keV}$ where the HB11 fusion reaction cross-section is exceptionally high producing three new alpha particles of $2.89 \mathrm{MeV}$ each. This is important for spherical compression where a density increase was also evaluated by hydrodynamics from extending the earlier numerical codes. Further, additional ion collisions in the range between 100 and 600 $\mathrm{keV}$ will increase this multiplication of the HB11 reactions producing an avalanched reaction. These secondary reactions are considerably increasing the gain and also the initial ignition process at the ps laser pulse interaction. If all fuel would react this is expressed by a factor $F=1$. The reduction of the threshold of the energy flux density $E_{0}{ }^{*}$ may be not so strong.

Combining the result (1) for DT with the higher value for HB11 without the secondary reactions in view of the result for DT (Hora et al., 2008)

$$
E_{0}^{*}=\left(10^{8} \mathrm{~J} / \mathrm{cm}^{2}\right),
$$

a reduction by a factor $B>1$ takes into account the not yet known reduction of the ignition flux limit due to the secondary reactions.

$$
E_{\mathrm{r}}^{*}=E_{0}^{*} / B \quad(B>1) .
$$


The prediction of $B$ is rather preliminary and may be around 5 but the most pessimistic case would be $B=1$. The total HB11 fusion energy in a fuel sphere 2 of Figure 3 with radius $R$ in $\mathrm{cm}$ is

$$
E=5.95 \times 10^{11} R^{3} \quad \text { Joule }
$$

The minimum laser energy for producing the fusion flame is for the time $t$ (in ps)

$$
E_{\mathrm{L}}=4 R^{2} E_{r}^{*} /(B t)
$$

If the radius of the fuel sphere is $1 \mathrm{~mm}$ and the laser pulse duration $1 \mathrm{ps}$, the laser power has to be $12.5 / B$ EW. This shows that the radius $R$ has to be less than $\mathrm{mm}$, if the laser pulse has to be $1 \mathrm{EW}$ for the pessimistic $B=1$. The gain is

$$
G=474 B R t F
$$

Where $t$ is in ps.

As a first rough estimation, we assume that the laser energy produced by the ICAN spherical output of the laser pulse at the sphere 1 with a radius of $1 \mathrm{~m}$ may be $1 \mathrm{EW}$ for driving the $1 \mathrm{ps}$ laser pulse of MJ energy for igniting the fusion flame in the solid HB11 fuel 2. Using a factor $B=3$, the diameter of the sphere 3 has to be $0.560 \mathrm{~mm}$. The fusion energy from the reaction has then a maximum value of $69 \mathrm{MJ}$. If the reactor would work with a sequence of $20 \mathrm{~Hz}$, the total nuclear power produced is then $1.38 \mathrm{GW}$ in the produced alpha particles. This energy can be converted per slowing down of the alphas by electric fields up to about 1.4 MV such that with a minimum of losses, GW power (electric) can be produced.

It should be mentioned that a fiber laser ICAN with spherical fiber ends at a radius of 1 meter (Fig. 3) for Exawatt spherical converging laser pulses would consist of about 10 million thicker low cost polymer fibers (Fleming et al., 2001) instead of the thin glass fibers for telecommunication, providing ideal optical diffraction limit properties and CPR amplification with pulses of ps or shorter (Eggleton et al., 2011). This offers a solution for laser intensities of $10^{24}$ $\mathrm{W} / \mathrm{cm}^{2}$ or higher.

\section{SUMMARY}

Due to the high energy flux necessary for side-on ignition of HB11 for ultrahigh plasma block acceleration, spherical contracting laser pulses of EW power and 1 ps duration are needed for a $>15 \mathrm{~Hz}$ operation of a Gigawatt power station where the ICAN-fiber laser pulse system needs to have a spherical output diameter pulse of more than $2 \mathrm{~m}$ shrinking to the reactor core with a solid HB11 fuel pellet of $0.56 \mathrm{~mm}$ diameter for initiating the fusion reaction flame. The comparable high secondary reactions of the alpha particles produce an avalanche reaction of high efficiency. The spherical reactor core for the EW-ps laser pulses for initiating the reaction flame may lead to a fusion gain up to a factor 69 for a power station with Gigawatt (electric). Next topics of research focus on the secondary reaction of the generated alpha particles producing an avalanche process. The secondary reactions for determining the multiplication factor $B$ for the energy flux density needs to be evaluated. The aim is to produce nuclear energy with less dangerous radioactive radiation than from burning coal per generated energy free from primary generation of neutrons (Weaver et al., 1973; Hora et al., 2011). This gain of nuclear energy without radiation problem is the essential difference offered with HB11 fuel through the ICAN-fiber laser by nonlinear collective interaction in contrast to the thermal-pressure dominated way for laser driven fusion energy from deuterium-tritium fuel used by NIF or related schemes.

\section{REFERENCES}

Azechi, H., Mima, K., et al. (2011). Plasma physics and laser development for the fast-ignition realization experiment (FIREX) Project. Nucl. Fusion 49, 104024.

Badziak, J., Kozlov, A.A., Makowksi, J., Parys, P., Ryc, L., WoLowski, J., Woryna, E. \& VAnkov, A.B. (1999). Investigation of ion streams emitted from plasma produced with a high-power picosecond laser. Laser Part. Beams 17, 323-329.

Badziak, J., Glowacz, S., Jablonski, S., Parys, P., Wolowski, J. \& HorA, H. (2004). Production of ultrahigh-current-density ion beams by short-pulse laser-plasma interaction. Appl. Phys. Lett. 85, 3041-3043.

BobIN, J.L. (1974). Nuclear fusion reactions in fronts propagating in solid DT. In Laser Interaction and Related Plasma Phenomena (Schwarz, H. \& Hora, H., Eds.), Vol. 4B, 465-494. New York: Plenum Press.

CHu, M.S. (1972). Thermonuclear reaction waves at high densities. Phys. Fluids 15, 412-422.

Eggleton, B.J., Luther-Davies, B. \& Richardson, K. (2011). Chalcogenide Photonics. Nat. Photonics 5, 141-148.

Fleming, S.C., Van Eijkelenborg, M., Sceats, A. \& Basset, I. (2001). Polymer Optical Wave Guide, US-Patent of granted Australian Patent 2001272230.

Földes, I.B., Bakos, J.S., Gal, K., Juhasz, Z., Kedves, M.A., Kocsis, G., Szatmari, S. \& Veres, G. (2000). Properties of high harmonics generated by ultrashort UV laser pulses on solid surfaces. Laser Phys. 10, 264-269.

HaAn, S.W., LindL, J.A., et al. (2011). Point design targets, specifications, and requirements for the 2010 ignition campaign on the National Ignition Facility. Phys. Plasmas 18, 051001.

HAAn, S.W. (2010). in LI, Yuandi. Nuclear power without radioactivity. Highlights in Chemical Technology - Royal Society of Chemistry Publishing, 24 March 2010 for Energy and Environ. Sci. 2010, 3, 479-486.

Hora, H. (1981). Physics of Laser Driven Plasmas. New York: John Wiley, 179.

Hora, H. (2004). Developments in inertial fusion energy and beam fusion at magnetic confinement. Laser Part. Beams 22, $439-449$.

Hora, H. (2009). Laser fusion with nonlinear force driven plasma blocks: Thresholds and dielectric effects. Laser Part. Beams 27, 207-222. 
Hora, H. (2013). Extraordinary jump of increasing laser fusion gains experienced at volume ignition for combination with NIF experiments. Laser Part. Beams 31, 228-232.

Hora, H., Lalousis, P. \& Eliezer, S. (1984). Analysis of the inverted double-layers produced by nonlinear forces in laserproduced plasmas. Phys. Rev. Lett. 53, 1650-1652.

Hora, H., Azechi, H., Kitagawa, Y., Mima, K., Murakami, M., NaKaI, S., Nishihara, K., TaKabe, H., YamanaKa, C., YamanaKa, M. \& YamanaKa, T. (1998). Measured laser fusion gains reproduced by self-similar volume compression and volume ignition for NIF conditions. J.Plasma Phys. 60, 743-760.

Hora, H., Badziak, J., Boody, F., Höpfl, R., Jungwirth, K., Kralikova, B., Kraska, J., Laska, L., Parys, P., Perina, P., Pfeifer, K. \& Rohlena, J. (2002). Effects of picosecond and ns laser pulses for giant ion source. Opt. Commun. 207, 333-338.

Hora, H., Badziak, J., Read, M.N., Li, Yu-Tong, Liang, Tian-Jiao, Liu, Hong, Sheng, Zheng-Ming, Zhang, Jie, Osman, F., Miley, G.H., Zhang, Weiyan, He, Xianto, Peng, Hanscheng, Glowacz, S., Jablonski, S., Wolowski, J., Skladanowski, Z., Jungwirth, K., Rohlena, K. \& Ullschmied, J. (2007). Fast ignition by laser driven particle beams of very high intensity. Phys.Plasmas 14, 072701/1-7.

Hora, H., Malekynia, B., Ghoranneviss, M., Miley, G.H. \& He, X. (2008). Twenty times lower ignition threshold for laser driven fusion using collective effects and the inhibition factor. Appl. Phys. Lett. 93, 011101/1-3.

Hora, H., Miley, G.H., Ghoranneviss, M., Malekynia, B. \& Azizi, N. (2009). Laser-optical path to nuclear energy without radioactivity: Fusion of hydrogen-boron by nonlinear forced driven plasma blocks. Optics Communications 282, 4124-4126.

Hora, H., Miley, G.H., Yang, X. \& Lalousis, P. (2011). Strong shock-phenomena at petawatt-picosecond laser side-on ignition fusion of uncompressed hydrogen-boron11. Astrophys. Space Sci. 336, 225-228.

Hora, H. \& Miley, G.H. (2011). Possibility for Gaining Nuclear Energy without Radioactivity from Solid Density Hydrogen Boron Using Lasers with Nonlinear Force Driven Plasma Blocks. J. Ener. Power Engin. 5, 718-729.

Hora, H., Sadighi-Bonabi, R., Yazdani, A., Afaideh, F., Nafari, F. \& Ghoranneviss, M. (2012). Effect of quantum correction on the acceleration and delayed heating of plasma blocks. Phys. Rev. E 85, 036404/1-6.

Hora, H., Lalousis, P., Moustaizis, S., Miley, G.H., Yang., X., He, X. T., Eliezer, S. \& Martinez-Val, J.-M. (2013). Shock Studies in Nonlinear Force Driven Laser Fusion with Ultrahigh Plasma Block Acceleration. IAEA-Fusion Conf. San Diego, CA., 2012 a Proceedings. IFEP602.pdf, 8 pages.

Kaluza, M., Schreiber, J., Sandala, M.I.K., Tsakiris, G.D., EIDmann, K., Meyer-Ter-Vehn, J. \& Witte, K. (2004). Influence of the laser prepulse on proton acceleration in thin foil experiments. Phys. Rev. Lett. 93, 045003.

Kouhi, M., Ghoraneviss, M., Malekynia, B., Miley, G.H., Sari, A. H., Azizi, N. \& Razavpour, S.S. (2011). Resonance effect for strong increase of fusion gains at thermal compression for volume ignition of Hydrogen Boron-11. Laser Part. Beams 29, 125-134.

Labaune, C., Depierraux, S., Goyon, C., Loisel, G., Yahia, V. \& RAFELSKI, J. (2013). Fusion reactions initiated by laseraccelerated particle beams in a laser-produced plasma Nature Commun. 4, 2506 doi:10.1038/ ncomms3506.

Lalousis, P. \& Hora, H. (1983). First direct electron and ion fluid computation of high electrostatic fields in dense inhomogeneous plasmas with subsequent nonlinear laser interaction, Laser and Particle Beams 1, 283-304.

LAlousis, P., FöldEs, I. \& HorA, H. (2012). Ultrahigh acceleration of plasma by picsoecond terawatt laser pulses for fast ignition of laser fusion. Laser and Particle Beams 30, 233-243.

Lalousis, P., Hora, H., Eliezer, S., Martinez-Val, J.-M., MoustizIS, S., Miley, G. H. \& Mourou, G. (2013). Shock mechanisms by ultrahigh laser accelerated plasma blocks in solid density targets for fusion. Phys. Lett. A 377, 885-888.

LindL, J.D. \& Moses, E.I. (2011). Plans for the national ignition campaign (NIC) on the national ignition facility (NIF): On the threshold of initiating ignition experiments. Phys. Fluids 18, 050901.

MaLeKynia, B. \& RaZAVIPouR, S.S. (2013). The internal propagation of fusion flame with strong shock of a laser driven plasma block for advanced nuclear fuel ignition. Chinese Phys. B 42, 055202.

Mourou, G. (2013). Summary Lecture. IZEST-LLNL Conference, Livermore, CA. 18 July 2013.

Mourou, G., Brlocklesby, B., Tajima, T. \& Limpert, J. (2013). The future is fibre accelerators. Nature Photon. 7, 258-261.

Moustaizis, S., Lalousis, P. \& Hora, H. (2013). A LIF scheme for HiPER application based on the combination of ultrahigh laser nonlinear force driven plasma blocks and the relativistic acceleration of ion blocks. In High Power, High Energy and HighIntensity Laser Technology and Research Using Extreme Light: Entering New Frontiers with Petawatt-Class Lasers (Hein, J., Korn, G. \& Silva, L.O., eds.) Proceedings of SPIE Vol. 8780, paper 878029 /1-10.

Sauerbrey, R. (1996). Acceleration of femtosecond laser produced plasmas. Phys. Plasmas 3, 4712-4716.

Stening, R.J., Khoda-Bakhsh, R., Pieruschka, P., Kasotakis, G., Kuhn, E., Miley, G.H. \& Hora, H. (1992). Laser Interaction and Related Plasma Phenomena (Miley, G.H. \& Hora, H., eds.), Vol. 10, p. 347. New York: Plenum Press.

Strickland, D. \& Mourou, G. (1985). Compression of amplified chirped optical pulses. Opt. Commun. 56, 219-221.

TAHIR, N.A. \& Hoffmann, D.H.H. (1997). Development of advanced inertial fusion targets. Laser Part. Beams 15, 575-587.

Weaver, T., Zimmerman, G. \& Wood, L. (1973). Exotic CTR fuel: Non-thermal effects and laser fusion application, Report UCRL74938, Livermore, CA: Lawrence Livermore Laboratory.

Zhang, P., He, J.T., Chen, D.B., Li, Z.H., Zhang, Y., Wong, Lang, LI, Z.H., Feng, B.H., Zhang, D.X., Tang, X.W. \& Zhang, J. (1998). X-ray emission from ultraintense-ultrashort laser irradiation. Phys. Rev. E 57, 3746-3752. 